\title{
Técnica de implante subpeitoral para tratamento de infecção de loja de marcapasso: estudo inicial
}

\author{
Acrisio Sales VALENTE *, Marcelo de Castro POCHINI", Ana Maria Rocha PINTO*, \\ Valquíria P. CAMPAGNUCCI ${ }^{*}$, Itagiba MARINELLI*, Sylvio Matheus de Aquino GANDRA*, \\ Luiz Antônio RIVETTI*
}

RBCCV 44205-529

\begin{abstract}
Valente A S, Pochini M C, Pinto A M R, Campagnucci V P, Marinelli I, Gandra S M A, Rivetti L A - Técnica de implante subpeitoral para tratamento de infecção de loja de marcapasso: estudo inicial. Rev Bras Cir Cardiovasc 2001; 16(1): 49-52.
\end{abstract}

RESUMO: Introdução/Objetivo: O implante de marcapasso cardíaco definitivo é um procedimento caracterizado por um baixo índice de complicações. No entanto, a infecção, principalmente a de loja de fonte geradora, caracteriza-se como uma das complicações mais comuns, com incidências variando entre 1-5\% na maioria dos centros. Diversas abordagens terapêuticas para o tratamento desta afecção vêm sendo descritas com resultados controversos. Observa-se, entretanto, uma tendência a melhores resultados, com menores índices de falência de tratamento ou reinfecção, nas abordagens mais agressivas, estas sempre relacionadas à alta morbidade e a altos custos finais. Com base nos princípios já bem descritos do poder bactericida dos flaps musculocutâneos associados à antibioticoterapia na esterilização de áreas pouco vascularizadas infectadas, foi desenvolvida e avaliada neste estudo inicial a técnica de implante subpeitoral para o tratamento dos casos de infecção restrita de loja de fonte geradora, objetivando-se menores morbidades e custos com, bons resultados.

Casuística e Método: Foi considerado no presente estudo o período de janeiro de 1996 a março de 2000 onde foram realizados 574 procedimentos, entre implantes e trocas de fontes geradoras, na Santa Casa de São Paulo, com um índice de infecção de 1,11\% (6 casos) e 2 casos de extrusão de fonte geradora sem infecção aparente. Quatro casos tratavam-se de infecção exclusiva de loja de fonte e em 2 casos houve comprometimento sistêmico com sepse. Os germes infectantes foram S.aureus, S.epidermidis e Pseudomonas. Foi utilizada como abordagem terapêutica para todos os casos sem comprometimento sistêmico a técnica de implante subpeitoral da mesma fonte geradora associada à antibioticoterapia específica.

Resultados: Não houve óbitos, casos de reinfecção ou falência de tratamento nos pacientes submetidos à técnica. O tempo médio de internação foi 7,3 dias. O tempo médio de antibioticoterapia foi de 7 dias. Não houve distúrbios do marcapasso que exigissem reabordagem. O seguimento é de 5 meses a 4 anos.

Conclusão: A técnica avaliada mostra-se, a princípio, como uma alternativa viável no tratamento da infecção de loja de marcapasso, com baixa morbidade e grande eficácia.

DESCRITORES: Estimulação cardíaca artificial. Marcapassos cardíacos, implante. Marcapassos cardíacos, efeitos adversos. Marcapasso artificial, efeitos adversos. Infecções bacterianas, terapia.

\footnotetext{
Trabalho realizado na Faculdade de Ciências Médicas da Santa Casa de São Paulo (FCMSCSP). São Paulo, SP, Brasil.

Recebido para publicação em junho de 2000.

* Da Faculdade de Ciências Médicas da Santa Casa de São Paulo (FCMSCSP)

Endereço para correspondência: Acrisio S. Valente. Rua Marjorie Prado, 112. São Paulo, SP, Brasil. CEP 04663-080. Tel. (11) 523-9479. e-mail: asvalente@osite.com.br.
} 
Valente A S, Pochini M C, Pinto A M R, Campagnucci V P, Marinelli I, Gandra S M A, Rivetti L A - Técnica de implante subpeitoral para tratamento de infecção de loja de marcapasso: estudo inicial. Rev Bras Cir Cardiovasc 2001; 16(1): 49-52.

\section{INTRODUÇÃO}

O implante de marcapasso definitivo é um procedimento caracterizado por um baixo índice de complicações. Entretanto, a infecção, principalmente a de loja de fonte geradora, apresenta-se como uma das complicações mais comuns, com incidências variando entre 1 a $5 \%$ na maioria dos centros (1) (Toronto General Hospital - 5,4\% (2); Wright State University - 4,0\% ${ }^{(3)}$; Catholic University Santiago - 1,3\% (4); Henry Ford Hospital - 1,06\% (5); Hospital Distrital de Faro - 6,9\% (6); Santa Casa de São Paulo - 1,03 \%).

Apesar de uma baixa incidência, as infecções merecem consideração especial por terem uma evolução potencialmente fatal, com alta morbidade e altos custos relacionados ao seu tratamento.

Diversas condutas têm sido descritas para o tratamento da infecção de loja de marcapasso. As abordagens terapêuticas mais conservadoras parecem cursar com maiores índices de recidiva ou falência de tratamento (6-10), sendo os melhores resultados relacionados às abordagens mais agressivas, com remoção completa e implante de novo sistema, conduta esta geralmente associada à alta permanência hospitalar e a altos custos.

O objetivo do presente estudo é demonstrar uma nova alternativa no manejo da infecção de loja de marcapasso, sem retirada ou implante de novo sistema, com baixa morbidade, menores custos e com bons resultados finais.

\section{CASUÍSTICA E MÉTODO}

Foram considerados no presente estudo 574 pacientes submetidos a implante ou troca de fonte geradora de marcapasso (325 implantes e 249 trocas de fonte geradora) no período de janeiro de 1996 a março de 2000 na Santa Casa de São Paulo. A média etária foi de 63,10 anos (1 dia de vida 94 anos) com $51,6 \%$ do sexo feminino e $48,4 \%$ do sexo masculino. Destes, 6 evoluíram com infecção de marcapasso $(1,11 \%), 4$ implantes e 2 trocas de fonte geradora, 4 homens e 2 mulheres com média etária de 63,3 anos (25 - 81 anos). Houve 2 casos de extrusão de fonte sem infecção aparente. Os germes infectantes foram S.aureus em 4 casos, S.epidermidis em 1 caso e Pseudomonas aeruginosa em outro caso. A manifestação da infecção ocorreu após 14 dias do procedimento em 2 casos, 5 meses em 1 caso, 6 meses em 1 caso, 11 meses em 1 caso e 3 anos em outro caso. Quatro pacientes apresentavam infecção somente da loja da fonte geradora com hemoculturas negativas e ecotransesofágico sem evidência de vegetações. Os germes descritos foram identificados através de cultura de secreção local. Um destes doentes apresentou febre e 2 apresentaram hemograma infeccioso. Houve 2 casos de infecção com sepse. Todos os pacientes haviam sido submetidos à antibioticoprofilaxia com cefalosporina de primeira geração por 48 horas após o procedimento inicial.

A abordagem terapêutica utilizada foi o implante subpeitoral da mesma fonte geradora no mesmo tempo cirúrgico associado à antibioticoterapia específica baseada em antibiograma nos 4 casos de infecção de loja. A mesma técnica foi utilizada no caso de extrusão da fonte (sem infecção aparente). Os casos com comprometimento sistêmico foram conduzidos com antibioticoterapia endovenosa e retirada da fonte geradora com exteriorização do cabo-eletrodo que foi conectado a uma fonte geradora externa. Um paciente evoluiu com resolução do quadro infeccioso, sendo retirado então o caboeletrodo existente e implantado um novo sistema contralateral. O outro paciente com comprometimento sistêmico evoluiu desfavoravelmente apesar da antibioticoterapia agressiva e posterior retidada do cabo endocárdico, falecendo no oitavo dia de internação.

\section{Técnica de Implante Subpeitoral}

O procedimento foi realizado no centro cirúrgico, com anestesia geral e intubação orotraqueal em 4 casos e anestesia local com sedação leve em 2 casos. Todo o tecido desvitalizado foi desbridado e a loja foi lavada com soro fisiológico, Povidine e Rifocina, assim como a fonte geradora. Posteriormente, o músculo peitoral maior foi divulsionado e o gerador reposicionado entre os músculos peitoral maior e menor. A seguir, o fechamento por planos sem nenhum tipo de drenagem (Figuras 1,2,3).

\section{RESULTADOS}

Não houve óbitos no período de internação hospitalar para os casos de infecção exclusiva de loja submetidos à técnica. O tempo médio de internação foi de 7,3 dias nos casos de implante subpeitoral e 30 dias no caso em que foi necessária a remoção com implante de novo sistema. Não houve recidivas infecciosas (controle com ecodopler e exames laboratoriais). Não se mostrou necessário o prolongamento da antibioticoterapia por tempo superior a 7 dias nos casos de implante subpeitoral (aval, clínica e laboratorial). Nenhum paciente apresentou qualquer distúrbio de funcionamento do marcapasso que exigisse reabordagem. Somente 1 paciente apresentou estimulação de loja no modo 
Valente A S, Pochini M C, Pinto A M R, Campagnucci V P, Marinelli I, Gandra S M A, Rivetti L A - Técnica de implante subpeitoral para tratamento de infecção de loja de marcapasso: estudo inicial. Rev Bras Cir Cardiovasc 2001; 16(1): 49-52.

unipolar, revertida com reprogramação para o modo bipolar. O seguimento é de 5 meses a 4 anos.

\section{COMENTÁRIOS}

As terapêuticas empregadas no tratamento da infecção de um sistema de marcapasso cardíaco artificial são diversas. No entanto, alguns aspectos sempre devem ser considerados na escolha da abordagem mais adequada. Nos casos de comprometimento sistêmico com sepse ou endocardite $(0,1$ $-2 \%$ ), ainda há uma tendência às abordagens mais agressivas com antibioticoterapia endovenosa prolongada (4 - 12 semanas) e remoção completa do sistema com implante de um novo geralmente em região contralateral (7-11). Nos casos de infecção exclusiva de loja ou de extrusão de fonte sem infecção aparente, algumas tentativas mais conservadoras vêm sendo estudadas mas ainda com taxas de insucesso e reinfecção maiores do que nas abordagens mais agressivas $(6,8,10)$. O germe infectante é outro aspecto considerado em alguns estudos que sugerem que condutas mais conservadoras possam ser tentadas nos casos de bactérias de menor virulência, como o S.epidermidis mas, nos casos de infecção por agentes de maior virulência como o $S$.aureus dentre outros, uma abordagem mais agressiva tem sido a aconselhada ${ }^{(5,12)}$. De uma maneira geral, as opções terapêuticas são várias, podendo ser citadas como mais comuns a antibioticoterapia associada ao reposicionamento do mesmo gerador $(6,8,10)$; antibioticoterapia associada à remoção completa do sistema com implante de um novo (7-11); antibioticoterapia associada a medidas locais e irrigação fechada (5), dentre outras. Todas estas associadas a longos períodos de internação, altos custos e resultados controversos.

Quando se consideram os casos de infecção de loja de fonte geradora, as terapêuticas empregadas também são variadas, dividindo-se em sua maioria entre as já descritas. Os resultados também ainda são bastante controversos, com os menores índices de recidiva sendo atribuídos às abordagens mais agressivas, estas cursando com longos períodos de internação hospitalar e altos custos.

Estimulados pela favorável experiência com uso de flaps musculocutâneios no controle de infecções mediastinais, procuramos utilizar o mesmo princípio no desenvolvimento de uma técnica alternativa para o tratamento da infecção de loja de marcapasso. O uso de tecidos altamente vascularizados, principalmente retalhos musculares, como auxiliar no tratamento de processos infecciosos estão bem descritos, como no revolucionário estudo de JEEVANANDAN (13) sobre a utilização de flaps musculocutâneos de musculatura peitoral para 0 tratamento da mediastinite, onde demonstra que as propriedades bactericidas do tecido muscular proporcionadas pela sua rica vascularização e atividade antigênica ${ }^{(14)}$ associadas a antibióticos apropriados promovem a esterilização do mediastino anterior. A técnica estudada por Jeevanandan consiste na reabertura do externo, desbridamento do tecido desvitalizado, lavagem local, dissecção da musculatura peitoral separando-a do gradeado costal até a linha axilar anterior e fechamento, no mesmo tempo cirúrgico, após o posicionamento de dois drenos laterais e um central, mantendo-se o esterno aberto e promovendo-se o avanço dos flaps de musculatura peitoral sobre a área infectada.Com esta técnica, o autor obteve altos índices de cura assim como diminuição do tempo de internação hospitalar e menor duração da antibioticoterapia. Também são de grande valia os estudos de CALDERON (14) e GOSAIN et al. (15) sobre o poder bactericida proporcionado pelos leucócitos liberados pelo fluxo sangüíneo dos flaps musculocutâneos observado em cães após testes de inoculação bacteriana. Desta forma, com base nestes princípios e com o intuito de encontrar uma alternativa terapêutica à infecção de loja de fonte geradora de marcapasso, com menores custos, morbidade e bons resultados finais, foi desenvolvida a técnica de implante subpeitoral avaliada neste estudo inicial.

\section{CONCLUSÃO}

A técnica de implante subpeitoral mostra-se, a princípio, como uma alternativa viável para o tratamento da infecção da loja de fonte geradora de marcapasso. Apresentou, no presente estudo, uma eficácia de $100 \%$, isto associado a um curto período de internação e, conseqüentemente, a um menor custo final se comparada às diversas experiências até agora descritas. Um maior seguimento, no entanto, ainda é necessário para uma conclusão definitiva. 
Valente A S, Pochini M C, Pinto A M R, Campagnucci V P, Marinelli I, Gandra S M A, Rivetti L A - Técnica de implante subpeitoral para tratamento de infecção de loja de marcapasso: estudo inicial. Rev Bras Cir Cardiovasc 2001; 16(1): 49-52.

RBCCV 44205-529

Valente A S, Pochini M C, Pinto A M R, Campagnucci V P, Marinelli I, Gandra S M A, Rivetti L A - Subpectoral pacemaker implantation technique for treatment of generator pocket infection: initial study. Rev Bras Cir Cardiovasc 2001; 16(1): 49-52.

ABSTRACT: Introduction: Permanent pacing implantation is a very low risk procedure. However, infectious complications, specially in the pacemaker generator pocket, are the most common one with incidences (1 to $5 \%$ ). These patients have been treated by many different therapeutic proceduros with controversial results. It has been well demonstrated that eradication of the infection is extremely difficult and the best results are reached through radical surgery consisting of removal of the pacemaker generator and electrode associated to antibiotic therapy. These procedures are related to high morbidity and expense. Muscle flaps are thought to have the ability to survive a bacterial inoculation and control infection. It also brings a rich network of blood supply to an infected and poorly vascularized area. Based in these facts we developed a new technique for the treatment of the generator pocket infection that is the subpectoral pacemaker implantationn.

Material and Methods: Between January 1996 and March 2000, a total of 574 pacemakers were implanted or reimplanted at Santa Casa de São Paulo. Infection at the site of implantation developed in 6 instances (incidence of 1.11\%) and two extrusions of generator without apparent infection. Four patients had infection involving only the pacemaker pocket and two patients had sepsis. The organisms cultured were Staphylococcus aureus, Streptococcus epidermis and Pseudomonas. At pacemaker pocket infection instances and in the pacemaker extrusion the patients were treated by this new approach associated to antibiotic therapy.

Results: There were no deaths, reinfection or failure of treatment. The mean hospital stay was 7.3 days and antibiotic therapy 7 days. The follow up is five months to four years.

Conclusions: The subpectoral pacemaker implantation technique is a good alternative for cases of pocket generator infection with low morbidity and good finals results.

DESCRIPTORS: Cardiac pacing, artificial. Cardiac stimulation, artificial.Cardiac pacemakers implantation. Cardiac pacemakers, adverse effects. Pacemakers, artificial, adverse effects. Bacterial infections, therapy.

\section{REFERÊNCIAS BIBLIOGRÁFICAS}

1 Hayes D - Causative organisms and predisposing factors of infection in permanent pacing systems. Pacing Clin Electrophysiol 1986; 4: 216-22.

2 Goldman BS - Commentary for Salvage of infected cardiac pacemaker pocket using a closed irrigation system. Pacing Clin Electrophysiol 1986; 9: 915-16.

3 Beeler BA - Infection of permanent trasvenous and epicardial pacemakers in adults. Heart Lung. 1982;11: 152-6.

4 Dubernet $\mathrm{J}$ - Surgical removal of entrapped endocardial pacemaker eclectrodes. Progr Pacing Eletrophysiol 1986; 4: 147-52.

5 Jara F M, Toledo Peruyra L, Lenvis JW, Magilligan DJ The infected pacemaker pocket. J Thorac Cardiovasc Surg 1979;

6 Jesus I \& Leiria G - The pocket infection-erosion of permanent pacemakers: the results of a conservative appraach without substitution of the components. Rev Port Cardiol 1995; 14: 691-5.

7 Antinori C H, Villanueva DT, Pierucci L, Manuele VJ, Kuechler JA - A new approach to the management of infected pacemakers. Clin Cardiol 1994; 17: 38-40.

8 Harjula A, Jarvinea A, Virtanen KS, Mattila S - Pacemakers infections: treatment with total or partial pacemaker system removal. Thorac Cardiovasc Surg 1985; 33: 218-20.
9 Griffith M J, Mounsey JP, Bexton RS, Holden MP Mechanical, but not Infective, pacemaker erosion may be successfully managed by re-implatation of pacemakers. Br Heart J 1994; 71: 202-5.

10 Molina J E - Undertreatment and overtreatment of patients with Infected antiarrhythmic implantable devices. Ann Thorac Surg 1997; 63: 504-9.

11 Da Costa A, Kirkorian G, Chevalier P et al. - Infections secondary to implantation of cardiac pacemakers. Arch Mal Coeur Vaiss 1998; 91: 753-7.

12 Ruiter J H, Degener JE, Van Mechelen R, Bos R - Late purulent pacemaker pocket infection caused by Staphylococcus epidermides: serious complications of in situ management. Pacing Clin Eletrophysiol 1985; 8: $903-7$

13 Jeevanandan $\mathrm{V}$ - Single-stage management of sternal wound infections. J Thorac Cardiovasc Surg 1990; 99: 256-63.

14 Calderon W, Shang N, Mathes SJ - Comparison of the effect of bacterial inoculation in musculocutaneous and Fascio-cutaneous flaps. Plast Reconstr Surg 1990; 77: 785-94.

15 Gosain A, Chang N, Mathes S, Hunt TK, Vasconez L A study of the relationship between blood flow and bacterial inoculationin in musculocutaneous and fasciocutaneous flaps. Plast Reconst Surg, 1990; 86: 1152-62. 